\title{
Abdominal paracentesis: use of a standardised pro forma improves patient safety with good record keeping
}

\author{
Authors: Md Deloar Hoshen ${ }^{\mathrm{A}}$ and Usama Mahmood ${ }^{\mathrm{A}}$
}

\section{Introduction}

Paracentesis, the aspiration of fluid from the abdominal cavity, is a diagnostic and therapeutic procedure frequently performed in gastroenterology wards. Paracentesis is generally a safe procedure but there are variations in practice and some potential complications such as bleeding and injury to internal organs. These potentially serious risks can be reduced by using safety checks and optimal technique. Following several near-miss incidents involving ascitic drains in our Trust, we undertook an audit cycle in order to improve patient safety.

\section{Materials and methods}

A total of 15 documentation components were identified as essential for safe ascitic drain insertion, management, and removal based on the current British Society of Gastroenterology guidelines ${ }^{1}$ and expert opinion. We audited a representative sample of 17 sets of patient medical notes who had undergone paracentesis on Ward 91 and 92 (gastroenterology wards) over a period of 6 weeks. Based on these results we designed a poster for junior doctors and a novel paracentesis pro forma in order to standardise the safety check and documentation process and facilitate best practice (Fig 1). Printed copies were made available on the wards with an electronic copy available on a shared drive. To assess its long-term effect and direct further work, two cycles of re-audit were completed over a period of 12 weeks after each intervention.

\section{Results and discussion}

The initial audit demonstrated that $100 \%$ of paracentesis entries included a record of verbal consent. However, there was a large variation in the quality of documentation, with an average of $47 \%(n=8)$ compliance with the essential documentation components. In particular, explanation of procedure-related complications, amount of local anaesthetic used, time of drain removal, total volume drained, and post-procedure care instructions were poorly recorded. In

Authors: ALeeds Teaching Hospitals NHS Trust, Leeds, UK

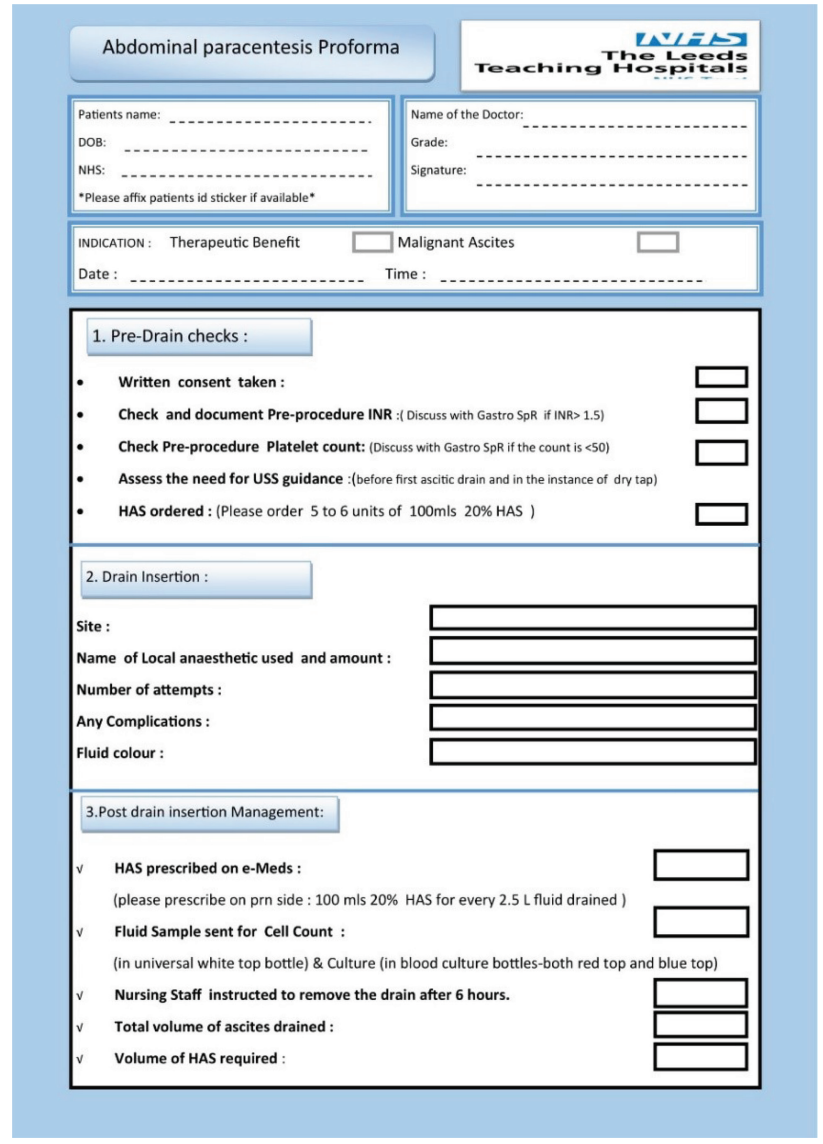

Fig 1. Paracentesis pro forma to standardise the safety check and documentation process.

order to encourage better compliance with the essential documentation components, we designed a poster for the doctors' office. Insignificant improvement was detected as a result of this intervention. However, the introduction of the paracentesis pro forma resulted in an immediate and sustained increase in documentation compliance to $83 \%$ $(\mathrm{n}=14)$. The greatest improvement was seen in recording preprocedure safety checks and post-procedure care instructions $(100 \% ; n=17)$. 


\section{Conclusion}

Introduction of a simple, standardised pro forma improves documentation in abdominal paracentesis, promoting best practice and safeguarding patient safety. It also boosts the confidence of junior doctors in performing the procedure.

\section{Conflicts of interest}

None declared.

\section{References}

1 Moore KP, Aithal GP. Guidelines on the management of ascites in cirrhosis. Gut 2006;55:1-12.

2. General Medical Council. Protecting children and young people: The responsibilities of all doctors. Keeping records. GMC, 2018. www.gmc-uk.org/ethical-guidance/ethical-guidance-for-doctors/ protecting-children-and-young-people/keeping-records 